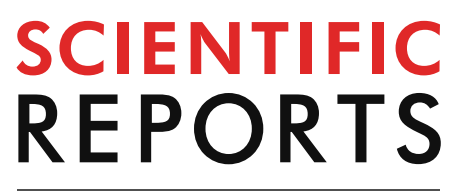

natureresearch

Check for updates

\title{
Determination of NUDT15 variants by targeted sequencing can identify compound heterozygosity in pediatric acute lymphoblastic leukemia patients
}

\author{
Chih-Hsiang Yu ${ }^{1}$, Ya-Hsuan Chang ${ }^{2}$, Der-Shiun Wang ${ }^{3,4}$, Shiann-Tarng Jou ${ }^{5}$, Chien-Yu Lin ${ }^{2}$, \\ Kai-Hsin Lin ${ }^{5}$, Meng-Yao Lu ${ }^{5}$, Lovely Raghav ${ }^{2}$, Hsiu-Hao Chang ${ }^{5}{ }^{5}$, Kang-Hsi Wu ${ }^{6}$, \\ Shu-Wei Chou ${ }^{5}$, Yu-Ling Ni ${ }^{7}$, Dong-Tsamn Lin ${ }^{5,7}$, Shu-Wha Lin ${ }^{1}$, Hsuan-Yu Chen ${ }^{2}$ \& \\ Yung-LiYang $\rrbracket^{5,7}$
}

Mercaptopurine intolerance is an adverse effect of mercaptopurine administration in pediatric acute lymphoblastic leukemia. Recently, NUDT15 variants were identified as a major determinant of mercaptopurine intolerance. Two NUDT15 variants, c.36_37insGGAGTC and c.415C > T, are located on exons 1 and 3, respectively. Patients with heterozygous c.36_37insGGAGTC and c.415C >T can be either compound heterozygous with two variants on different alleles or heterozygous with both variants on the same allele. Because patients with biallelic NUDT15 variants are extremely sensitive to mercaptopurine, clinical identification of NUDT15 diplotype would be advantageous. A cohort of 37 patients with c.36_37insGGAGTC and c.415C > T NUDT15 variants were selected for haplotyping by targeted sequencing. NUDT15 complementary DNA was amplified and sequenced by 300 -bp pairedend sequencing on Illumina MiSeq. Of the 37 patients carrying NUDT15 variants, 35 had heterozygous NUDT15*1/*2 variants and two had compound heterozygous NUDT15*3/*6 and NUDT15*2/*7 variants. These two patients with compound heterozygous variants could only tolerate low doses of mercaptopurine, similar to patients with homozygous NUDT15 variants. Targeted sequencing of NUDT15 CDNA can be used to determine NUDT15 diplotype and identify patients with compound heterozygous NUDT15 variants.

Thiopurines (e.g., 6-mercaptopurine [6-MP], 6-thioguanine [6-TG], and azathioprine [AZA]) are important antimetabolite drugs that are used for different clinical indications. In pediatric acute lymphoblastic leukemia (ALL), 6-MP is widely used in the induction, consolidation (with high-dose methotrexate), and maintenance phases. Indeed, 6-MP-based maintenance therapy is one of the most critical components in the treatment regimen for pediatric $\mathrm{ALL}^{1-7}$. Variants of genes responsible for thiopurine metabolism can directly influence drug toxicity and antileukemic efficacy ${ }^{8-13}$. For example, polymorphisms in the thiopurine methyltransferase (TPMT) gene have been linked to susceptibility to thiopurine-induced marrow suppression in patients, and preemptive TPMT genotype-guided dosing represents a successful example of genetics-based precision medicine in cancer treatment ${ }^{14,15}$.

\footnotetext{
${ }^{1}$ Departments of Clinical Laboratory Sciences and Medical Biotechnology, National Taiwan University, Taipei, Taiwan. ${ }^{2}$ Institute of Statistical Science Academia Sinica, Taipei, Taiwan. ${ }^{3}$ Department of Pediatrics, Tri-Service General Hospital, Taipei, Taiwan. ${ }^{4}$ Graduate Institute of Clinical Medicine, College of Medicine, National Taiwan University, Taipei, Taiwan. ${ }^{5}$ Department of Pediatrics, National Taiwan University Hospital and National Taiwan University College of Medicine, Taipei, Taiwan. ${ }^{6}$ Division of Pediatric Hematology and Oncology, China Medical University Children's Hospital, Taichung, Taiwan. ${ }^{7}$ Department of Laboratory Medicine, National Taiwan University Hospital and National Taiwan University College of Medicine, No. 7, Chung Shan S. Rd, Zhongzheng Dist, Taipei City 10041, Taiwan. ${ }^{\varpi}$ email: yangyl92@ntu.edu.tw
} 
NUDT15*1

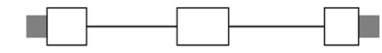

c.36_37insGGAGTC

NUDT15*2

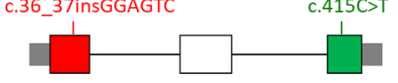

NUDT15*3

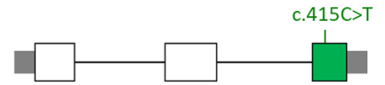

NUDT15*5

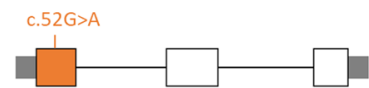

NUDT15*6

c.36_37insGGAGTC

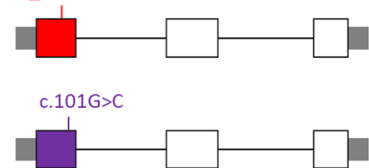

Figure 1. Four coding variants in NUDT15 were identified, representing six haplotypes (labeled as NUDT15*1-3 and NUDT15`5-7).

Recent genome-wide association studies have reported a missense variant in NUDT15 ( $r s 116855232$, referred to as c.415C > T or p.Arg139Cys) that is strongly associated with thiopurine-related myelosuppression in patients with inflammatory bowel disease in a Korean population ${ }^{16}$. In a genome-wide association study, Yang et al. identified this variant in association with mercaptopurine intolerance in childhood ALL ${ }^{17}$. Several NUDT15 variants associated with decreased diphosphatase activity have been reported, and haplotypes with different combinations of the variant(s) have been assigned star allele numbers, which were proposed by Moriyama et al. (Fig. 1) ${ }^{18,19}$. All haplotypes except for NUDT $15^{\star} 2$ have been reported to carry a single coding variant, including NUDT15*3 $(r s 116855232$, c.415C > T), NUDT15`5 (rs186364861, c.52G > A), NUDT15^6 (rs869320766, c.36_37insGGAGTC ), and NUDT15*7 (rs766023281, c.101G >C). NUDT15*2 carries both c.415C > T and c.36_37insGGAGTC, which are also present in $N U D T 15^{\star} 3$ and $N U D T 15^{\star} 6$, respectively. Numerous studies have established that NUDT15 variants are associated with mercaptopurine intolerance in pediatric ALL in different populations, highlighting the importance of preemptive genetic typing in these patients ${ }^{20-30}$.

Because of the strong correlation between the number of NUDT15 risk alleles and median 6-MP dosage, accurate determination of diplotypes might allow preemptive dosing ${ }^{31}$. For example, patients with heterozygous c. $415 \mathrm{C}>\mathrm{T}$ and c.36_37insGGAGTC can be determined to be either heterozygous (NUDT15*1/*2) or compound heterozygous (NUDT15*3/*6), and the tolerated 6-MP dosage by patients with the two diplotypes might be significantly different. In this study, we elucidated the NUDT15 diplotypes in patients with multiple heterozygous variants by next-generation sequencing (NGS) of NUDT15 complementary deoxyribonucleic acid (cDNA). Thus, this study will expand our knowledge of NUDT15 variants in patients with pediatric ALL in Taiwan.

\section{Methods}

Patients. We selected pediatric patients with ALL younger than 18 years of age treated in the National Taiwan University Hospital, Taipei, between April 1997 and December 2019. This study was approved by the Institutional Review Board of the National Taiwan University Hospital. Informed consent was obtained from the parents or legal guardians of the patients. This cohort was previously published ${ }^{30}$, and we selected a subset of patients for this study. There were 22 patients with heterozygous c.36_37insGGAGTC and c.415C > T variants, and 10 patients with known NUDT15 diplotypes (4 with $N U D T 15^{\star} 1 /{ }^{\star} 1,2$ with $N U D T 15^{\star} 1 /{ }^{*} 3,1$ with $N U D T 15^{\star} 1{ }^{\star} 5,1$ with $N U D T 15^{\star} 1 /^{\star} 6$, and 2 with $N U D T 15^{\star} 2 /^{\star} 2$ ) were enrolled in the previous study ${ }^{30}$. In this study, another 15 patients with heterozygous c.36_37insGGAGTC and c.415C $>$ T variants, 1 patient with $N U D T 15^{\star} 1 /{ }^{\star} 5$, and 1 patient with $N U D T 15^{\star} 2 / * 3$ were enrolled.

Targeted sequencing-based haplotyping. Forty-nine patient samples were selected for haplotyping by targeted sequencing, including 37 patients with heterozygous c.36_37insGGAGTC and c.415C $>$ T variants. Samples from 12 patients with known NUDT15 diplotypes $\left(N U D T 15^{\star} 1 /^{\star} 1, N U D T 15^{\star} 1{ }^{\star} 3, N U D T 15^{\star} 1{ }^{\star} 5\right.$, NUDT15*1/*6, NUDT15*2/*2, and NUDT15*2/*3) were included as experimental controls. Total RNA was extracted using TRIzol reagent according to the manufacturer's instructions (Invitrogen, Waltham, MA, USA). We synthesized cDNA from germline total RNA using the Maxima First-Strand cDNA Synthesis Kit (Thermo Fisher Scientific, Waltham, MA, USA). NUDT15 cDNA was amplified using the Phusion Hot Start II HighFidelity PCR Master Mix (Thermo Fisher Scientific) with barcoded primers (Tables S1 and S2). The barcoded amplicons were purified using AMPure XP beads (Beckman Coulter, Brea, CA, USA) and quantified using Qubit reagent (Thermo Fisher Scientific). Equimolar amounts of each amplicon were pooled and sequenced by 300-bp paired-end sequencing using the MiSeq Reagent Kit v3 (Illumina, San Diego, CA, USA). Raw sequencing data were further analyzed to discriminate the NUDT15 haplotypes. The barcoded primers used for the targeted sequencing of cDNA are listed in Supplementary Table 1.

FastQC (v0.11.7) was performed to evaluate the quality and adaptor contamination of the sequencing reads. Adaptor sequences and low-quality bases were trimmed (average quality score of a four-base wide sliding window <20) using Trimmomatic (v0.33). The trimmed reads were further aligned to NUDT15 cDNA (NM_018283) using bowtie 2 (v2.3.3.1). The average insert length of the reads was $562 \mathrm{bp}$. Reads with specific variants were extracted from the total aligned reads and used to determine the two alleles of NUDT15. Finally, IGV tool (v2.7.2) was used to check the read alignment results and the two alleles of the samples.

Cloning of NUDT15 cDNA. The NUDT15 cDNA amplicons were purified using the FavorPrep GEL/PCR Purification Kit (Favorgen Biotech, Ping-Tung, Taiwan), cloned into a vector using the Zero Blunt TOPO PCR 
Cloning Kit (Thermo Fisher Scientific), and transformed into TOP10 competent cells (Thermo Fisher Scientific). Single colonies were cultured in LB broth $\left(50 \mu \mathrm{g} / \mathrm{mL}\right.$ kanamycin) at $37^{\circ} \mathrm{C}$ for $16 \mathrm{~h}$, and plasmids were extracted using the FavorPrep Plasmid DNA Extraction Mini Kit (Favorgen Biotech). Sanger sequencing was performed using the SP6 or T7 primer (Supplementary Table 1). At least 20 plasmids from a single colony were sequenced to determine the NUDT15 diplotypes.

Statistical analyses. Mann-Whitney $\mathrm{U}$ test was used to evaluate differences in median 6-MP dosages between patients with a heterozygous variant $\left(N U D T 15^{\star} 1 /^{\star} 2\right)$ and homozygous/compound heterozygous variant $\left(N U D T 15^{\star} 2{ }^{*} 2, N U D T 15^{\star} 2{ }^{\star} 3, N U D T 15^{\star} 2 /{ }^{*}\right.$, and NUDT15*3/*6).

\section{Results}

Next-generation sequencing of cDNA. To resolve the diplotypes of patients with multiple heterozygous variants, we sequenced NUDT15 cDNA by paired-end sequencing. Thirty-seven patients had multiple heterozygous variants. We also included the cDNA of 12 patients with known diplotypes ( 4 with $N U D T 15^{\star} 1 /^{\star} 1,2$ with

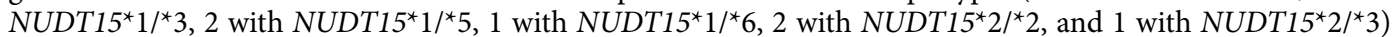
as experimental controls ${ }^{30}$. The reads were phased, and the results showed that all but two samples could be classified as $N U D T 15^{\star} 1 /^{\star} 2$ (Fig. 2a). The two samples with different allele combinations were classified as NUDT15*3/*6 (Fig. 2b) and NUDT15*2/*7 (Fig. 2c).

cDNA sequencing of the NUDT15*3/*6 patient. To validate the targeted sequencing result for the patient with $N U D T 15^{\star} 3 /^{\star} 6$, the NUDT15 cDNA was cloned into a vector, and the haplotypes were resolved by Sanger sequencing of the vector. The results showed that the c.36_37insGGAGTC and c.415C > T alleles were located in trans; thus, the genome of this patient was determined to harbor a compound heterozygous NUDT15*3/*6 variant (Figure S1). We also genotyped the biological parents of this patient and found that the genomes of the parents harbored $N U D T 15^{\star} 1 /^{*} 6$ and $N U D T 15^{\star} 1{ }^{*} 3$. The genotyping of the biological parents of patients provides an additional line of evidence to determine the diplotypes (Fig. 3 ).

CDNA sequencing of the NUDT15*2/*7 patient. We also validated the result of the patient with NUDT $15^{\star} 2 /^{\star} 7$ by cDNA cloning and showed that c.101G $>C$ was located in trans with c.36_37insGGAGTC and c.415C > T (Figure S2). The biological parents of the patients were genotyped, and we found that the father was homozygous for c.36_37insGGAGTC and c.415C $>\mathrm{T}\left(N U D T 15^{\star} 2 /^{\star} 2\right)$, whereas the mother was heterozygous for the c.36_37insGGAGTC, c.415C > T, and c.101G >C variants, which matched the genotype of the patient. Because the patient must have had an NUDT15 2 allele from the father, the c.101G $>C$ allele should have been inherited from the mother; we can posit that the diplotype of the mother is NUDT15*2/*7.

Tolerated doses of 6-MP among patients with the NUDT15 variant. We evaluated the median tolerated 6-MP dose in patients with the NUDT15 variants. The median tolerated 6-MP dose among all 35 patients with the NUDT15*1/*2 genotype was $12.5 \mathrm{mg} / \mathrm{m}^{2}\left(5.0-42.5 \mathrm{mg} / \mathrm{m}^{2}\right)$, and the doses were extremely low for the patients with $N U D T 15^{\star} 2 /^{\star} 2$ or NUDT15*2/*3 $\left(1,2.2\right.$, and $\left.4.3 \mathrm{mg} / \mathrm{m}^{2}\right)$. The two patients with compound heterozygous variants, NUDT1 $15^{\star} 3 /^{*} 6$ and $N U D T 15^{\star} 2{ }^{*} 7$, tolerated only 2.5 and $6.7 \mathrm{mg} / \mathrm{m}^{2} /$ day mercaptopurine, respectively, and this was similar to the patients with homozygous variants. We observed that patients with homozygous and compound heterozygous NUDT15 variants tolerated a significantly lower median 6-MP dose than patients with heterozygous variants (Fig. 4).

\section{Discussion}

Our study and that of Tsujimoto et al..$^{32}$ showed that most patients with the NUDT15 c.36_37insGGAGTC and c. $415 \mathrm{C}>\mathrm{T}$ variants harbored heterozygous NUDT15* $1 /^{\star} 2$. Tsujimoto et al. defined the diplotypes of 14 patients carrying the two variants (i.e., c.415C > T and c.36_37insGGAGTC) by droplet digital PCR (ddPCR) and restriction enzyme-PCR (RE-PCR ${ }^{32}$. We developed another method using NUDT15 cDNA-targeted sequencing. The NUDT15 cDNA was sequenced by paired-end sequencing using Illumina MiSeq, and our results were confirmed by cloning. Compared with ddPCR and RE-PCR, targeted sequencing of NUDT15 cDNA can help determine the diplotypes without genotyping information of a patient.

Currently, NUDT15 genotyping is indicated before mercaptopurine administration for pediatric ALL ${ }^{31}$. Poor metabolizers are defined as individuals carrying two nonfunctioning alleles $\left(N U D T 15^{\star} 2 /^{\star} 2, N U D T 15^{\star} 2 /^{\star} 3\right.$, and NUDT15*3/*3). Potential intermediate metabolizers are individuals carrying one allele of uncertain function plus one nonfunctioning allele, such as NUDT15 $2{ }^{\star} 5$ and NUDT15*3/* $6^{31}$. Our patient with the NUDT15 $3 /{ }^{\star} 6$ combination tolerated only $2.5 \mathrm{mg} / \mathrm{m}^{2} /$ day mercaptopurine, and this was similar to the dose for poor metabolizers. Another patient with NUDT15*2/*7 could also tolerate very low daily doses of mercaptopurine. The c. $101 \mathrm{G}>\mathrm{C}$ variant of NUDT15 is also very rare in Asians, and its function was confirmed by Moriyama et al ${ }^{19}$. From our experience with these two patients, any combination of two mutant alleles with known functional roles might be classified as compound heterozygous, and the mercaptopurine dose should be the same as that administered to homozygous patients. Because mercaptopurine is widely administered for other diseases, such as inflammatory bowel disease ${ }^{33,34}$, its clinical significance has also been validated in this subgroup of patients. The identification of these two compound heterozygous patients suggests that testing laboratories should be cautious while interpreting the c.36_37insGGAGTC and c.415C > T variants of NUDT15 in genetic testing of pediatric patients with ALL taking mercaptopurine or other related drugs, rather than only considering these genotypes. 
a

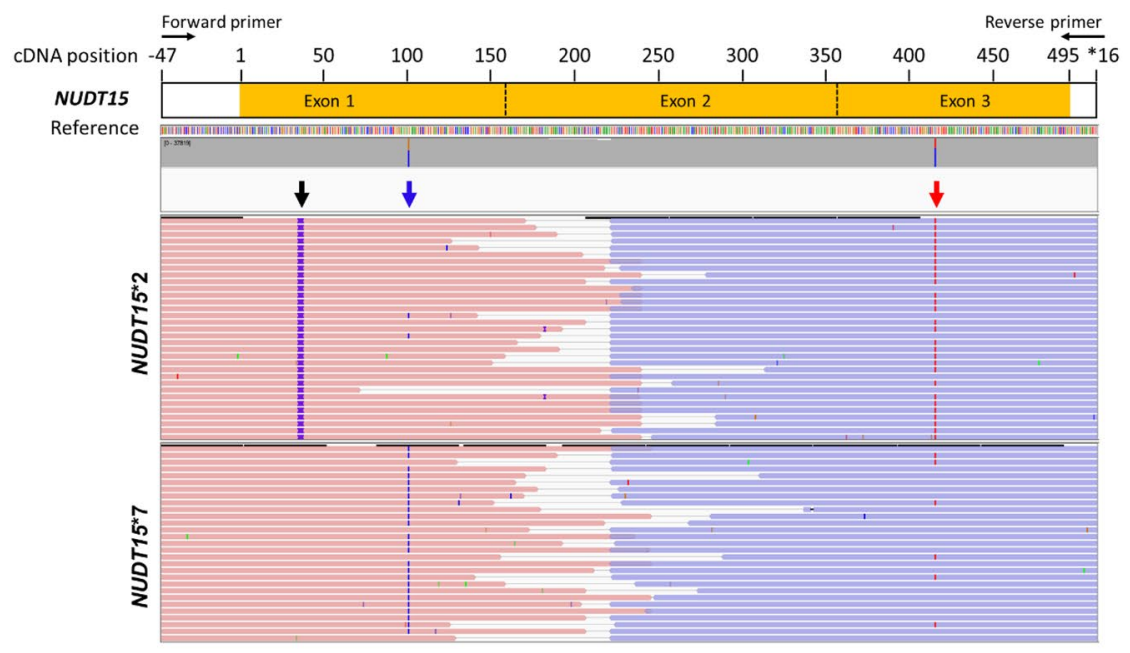

b

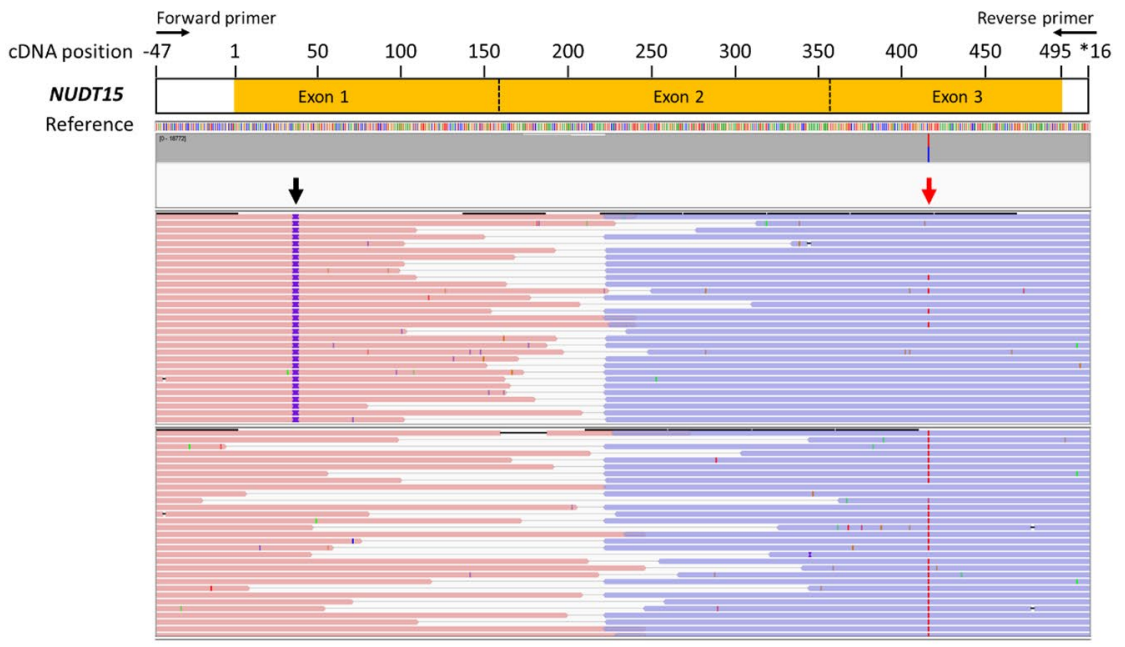

C

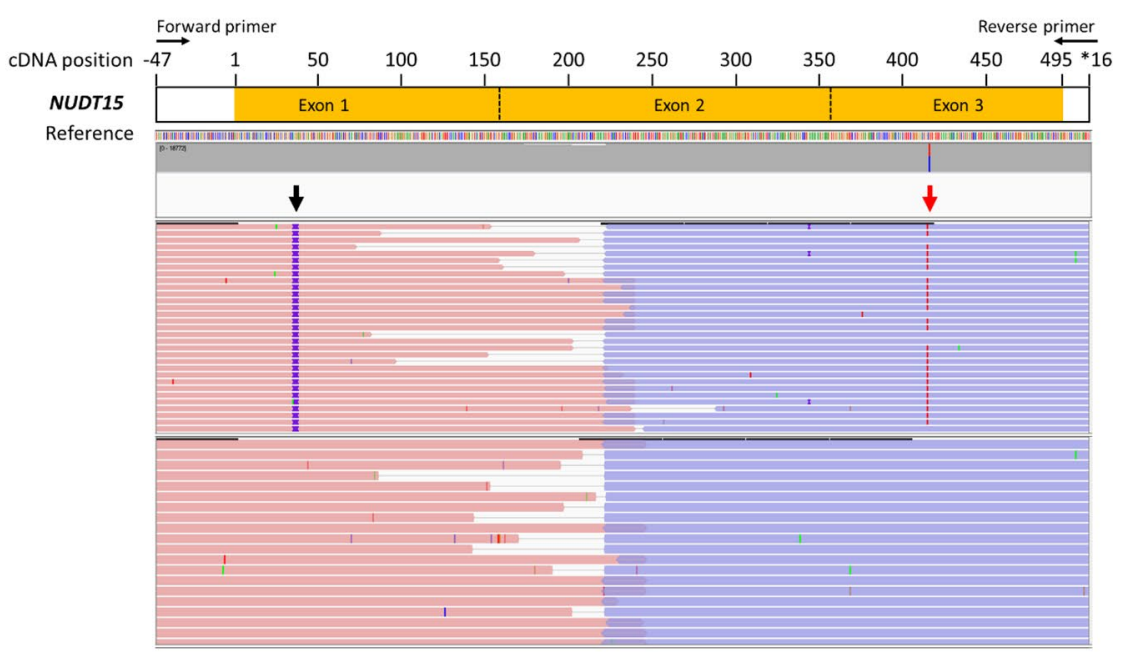

Figure 2. Phased variants of NUDT15. (a) Patients (NTUCH07) with NUDT15*1/*2, (b) patients (NTUCH27) with $N U D T 15^{\star} 3{ }^{*} 6$, and (c) patients (NTUCH37) with $N U D T 15^{\star} 2 /^{*} 7$ are shown. Reads were mapped to NUDT15 mRNA reference sequence (NM_018283), and cDNA position relative to gene structure is presented. The NUDT15 variants are indicated with arrows, and reads were grouped by haplotype for these variants. Black arrow: c.36_37insGGAGTC; blue arrow: c.101G > C; red arrow: c.415C > T. The paired-end reads are represented, and the reads are colored red for forward strand and blue for reverse strand. 
Exon 1

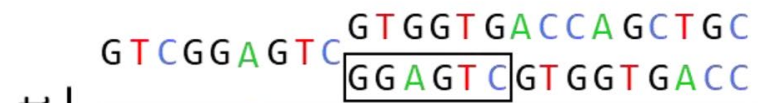

$\underset{\frac{n}{ \pm}}{\frac{ \pm}{\pi}}$

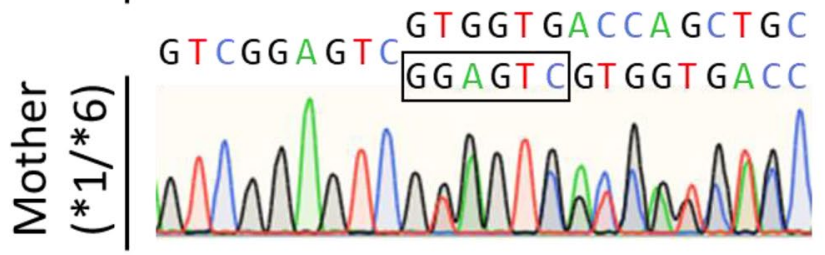

GTCGGAGTCGTGGTGACCA GCTGC

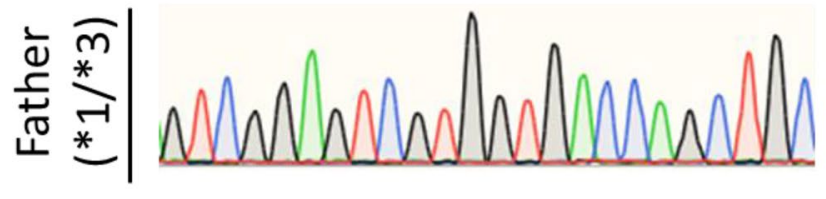

Exon 3

G GACTG ${ }_{T}^{\mathrm{C}} \mathrm{G} T \mathrm{TTTTTA}$

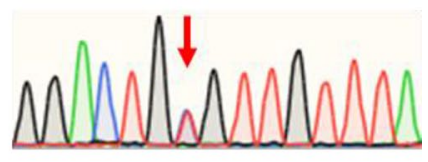

G GAC TGCG TTGTTTA

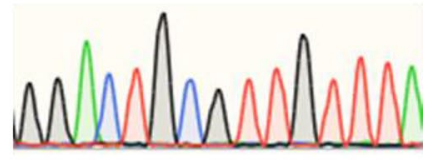

G GAC TG ${ }_{T}^{C}$ G TTGTTTA

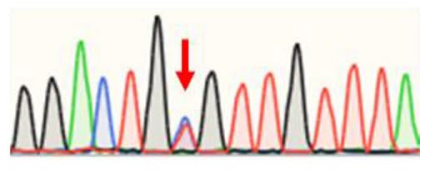

$$
\begin{aligned}
& \square: \text { c.36_37insGGAGTC } \\
& \longrightarrow: \text { c.415C>T }
\end{aligned}
$$

Figure 3. Patient with heterozygous c.36_37insGAGTCG and c.415C > T variants. We performed Sanger sequencing of the biological parents of the patient. The mother had a heterozygous c.36_37insGAGTCG variant $\left(N U D T 15^{\star} 1{ }^{*} 6\right)$ and father had a heterozygous c. $415 \mathrm{C}>\mathrm{T}$ variant $\left(N U D T 15^{\star} 1{ }^{\star} 3\right)$.

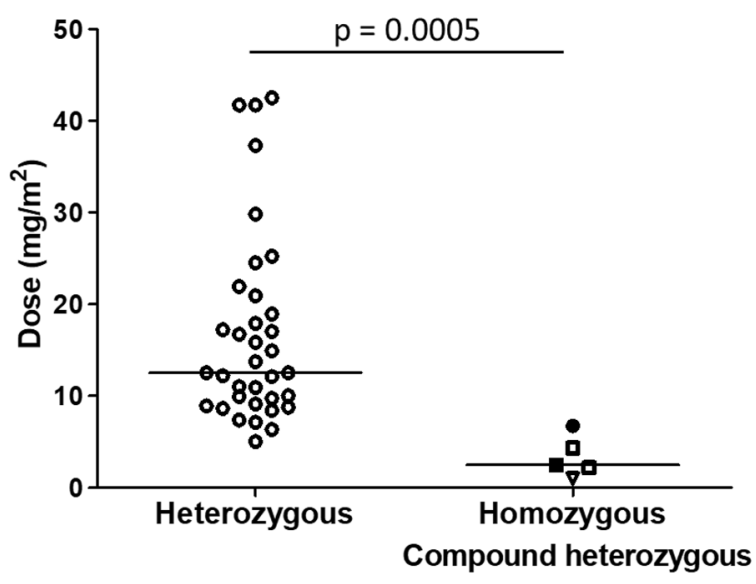

\begin{tabular}{lll} 
& NUDT15*2/*2 \\
Diplotypes & NUDT15*1/*2 & NUDT $2 / 5^{*} 3$ \\
& & NUDT15*3/*6 \\
& & NUDT15*2/*7 \\
\hline
\end{tabular}

Patient number

35

5

Figure 4. Distribution of tolerated 6-MP doses according to NUDT15 diplotypes. Heterozygous (open circles: NUDT15*1/*2), homozygous (open triangle: $N U D T 15^{\star} 2{ }^{*} 2$, open square: $N U D T 15^{\star} 2{ }^{*} 3$ ), or compound heterozygous (closed square: NUDT15*3/*6, closed circle: NUDT15*2/*7)) and patient number are represented. The P-value was calculated using Mann-Whitney U test. 
There was a major limitation to this study. From a cost and benefit perspective, it might be difficult to use cDNA NGS to determine the diplotype of NUDT15 in clinical practice; moreover, the incidence of compound heterozygous mutations, such as $N U D T 15^{\star} 3 /{ }^{*} 6$, is very $\operatorname{rare}^{18}$. Sanger sequencing remains the most convenient and cost-effective method for the analysis of genetic variants of NUDT15 $5^{17,18,20,25,27,28,31,32,35}$. However, to improve clinical outcomes and reduce adverse effects in pediatric patients with ALL, appropriate preemptive diagnosis of pharmacogenetic variants might be warranted ${ }^{31,36,37}$. There were other genetic polymorphisms related to some common adverse effects of chemotherapy, such as vincristine neuropathy, asparaginase hypersensitivity, hypertension, and osteonecrosis due to steroid use ${ }^{37-45}$. NGS cDNA targeted therapy might have some advantages, including sequencing multiple genes simultaneously and identifying the diplotype of NUDT15 variants if complex variants are present. If multiple genetic variants can be sequenced simultaneously, the cost might be reduced for individual SNPs and avoid tedious multiple Sanger sequencing. Although NUDT15 variants are very rare, identifying compound homozygous patients before the administration of mercaptopurine might largely reduce the initial dose of mercaptopurine to avoid profound marrow suppression. Thus, the infectious adverse effect might be decreased in this small subset of patients.

In conclusion, most c.36_37insGGAGTC and c.415C > T variants in NUDT15 are located on the same allele and can be classified as monoallelic variants. Although very rare, there are patients with compound heterozygous NUDT15 variants. Patients with any combination of functionally verified NUDT15 alleles might be considered to be poor mercaptopurine metabolizers, and these patients should be administered a relatively low dose of this drug. An appropriate definition of compound heterozygous variants in NUDT15 might require genotyping of the biological parents of the patient or other molecular methods, such as targeted sequencing, ddPCR, RE-PCR, and cloning.

Ethics declaration. This study was conducted in accordance with the Declaration of Helsinki guidelines. Written informed consentwas obtained from all study participants or their guardians. The study was approved by the Institutional ResearchBoard of National Taiwan University Hospital (NTUH IRB number 201510016RIND).

\section{Consent to participate. None.}

\section{Data availability}

Data of cDNA sequencing of NUDT15 have been deposited at https://www.ncbi.nlm.nih.gov/sra/PRJNA655987.

Received: 4 January 2020; Accepted: 17 August 2020

Published online: 01 September 2020

\section{References}

1. Vora, A. et al. Treatment reduction for children and young adults with low-risk acute lymphoblastic leukaemia defined by minimal residual disease (UKALL 2003): a randomised controlled trial. Lancet Oncol. 14, 199-209 (2013).

2. Alexander, T. B. et al. The genetic basis and cell of origin of mixed phenotype acute leukaemia. Nature 562, 373-379 (2018).

3. Pui, C. H. \& Evans, W. E. Treatment of acute lymphoblastic leukemia. N. Engl. J. Med. 354, 166-178 (2006).

4. Pui, C.-H. et al. Childhood acute lymphoblastic leukemia: progress through collaboration. J. Clin. Oncol. 33, 2938-2948 (2015).

5. Maltzman, J. S. \& Koretzky, G. A. Azathioprine: old drug, new actions. J. Clin. Invest. 111, 1122-1124 (2003).

6. Karran, P. \& Attard, N. Thiopurines in current medical practice: molecular mechanisms and contributions to therapy-related cancer. Nat. Rev. 8, 24-36 (2008).

7. Elion, G. The purine path to chemotherapy. Science 244, 41-47 (1989).

8. Kunz, J. B. et al. Pediatric T-cell lymphoblastic leukemia evolves into relapse by clonal selection, acquisition of mutations and promoter hypomethylation. Haematologica 100, 1442-1450 (2015).

9. Meyer, J. A. et al. Relapse-specific mutations in NT5C2 in childhood acute lymphoblastic leukemia. Nat. Genet. 45, 290-294 (2013).

10. Tzoneva, G. et al. Clonal evolution mechanisms in NT5C2 mutant-relapsed acute lymphoblastic leukaemia. Nature 553, 511-514 (2018).

11. Tzoneva, G. et al. Activating mutations in the NT5C2 nucleotidase gene drive chemotherapy resistance in relapsed ALL. Nat. Med. 19, 368-371 (2013).

12. Li, B. et al. Negative feedback-defective PRPS1 mutants drive thiopurine resistance in relapsed childhood ALL. Nat. Med. 21, 563-571 (2015).

13. Relling, M. V. et al. Mercaptopurine therapy intolerance and heterozygosity at the thiopurine S-methyltransferase gene locus. JNCI J. Natl. Cancer Inst. 91, 2001-2008 (1999).

14. Krynetski, E. Y. et al. A single point mutation leading to loss of catalytic activity in human thiopurine S-methyltransferase. Proc. Natl. Acad. Sci. USA 92, 949-953 (1995).

15. Relling, M. V. et al. Clinical pharmacogenetics implementation consortium guidelines for thiopurine methyltransferase genotype and thiopurine dosing: 2013 update. Clin. Pharmacol. Ther. 93, 324-325 (2013).

16. Yang, S. K. et al. A common missense variant in NUDT15 confers susceptibility to thiopurine-induced leukopenia. Nat. Genet. 46, 1017-1020 (2014).

17. Yang, J. J. et al. Inherited NUDT15 variant is a genetic determinant of mercaptopurine intolerance in children with acute lymphoblastic leukemia. J. Clin. Oncol. 33, 1235-1242 (2015).

18. Moriyama, T. et al. NUDT15 polymorphisms alter thiopurine metabolism and hematopoietic toxicity. Nat. Genet. 48, 367-373 (2016).

19. Moriyama, T. et al. Novel variants in NUDT15 and thiopurine intolerance in children with acute lymphoblastic leukemia from diverse ancestry. Blood 130, 1209-1212 (2017).

20. Buaboonnam, J. et al. Effect of NUDT15 on incidence of neutropenia in children with acute lymphoblastic leukemia. Pediatr. Int. 61, 754-758 (2019).

21. Choi, R. et al. Pathway genes and metabolites in thiopurine therapy in Korean children with acute lymphoblastic leukaemia. Br. J. Clin. Pharmacol. 85, 1585-1597 (2019).

22. Khera, S. et al. Prevalence of TPMT, ITPA and NUDT 15 genetic polymorphisms and their relation to 6MP toxicity in north Indian children with acute lymphoblastic leukemia. Cancer Chemother. Pharmacol. 83, 341-348 (2019). 
23. Liang, D. C. et al. NUDT15 gene polymorphism related to mercaptopurine intolerance in Taiwan Chinese children with acute lymphoblastic leukemia. Pharmacogenom. J 16, 536-539 (2016).

24. Moradveisi, B. et al. ITPA, TPMT, and NUDT15 genetic polymorphisms predict 6-mercaptopurine toxicity in middle eastern children with acute lymphoblastic leukemia. Frontiers Pharmacol. https://doi.org/10.3389/fphar.2019.00916 (2019).

25. Schaeffeler, E. et al. Impact of NUDT15 genetics on severe thiopurine-related hematotoxicity in patients with European ancestry. Genet. Med. https://doi.org/10.1038/s41436-019-0448-7 (2019).

26. Soler, A. M. et al. TPMT and NUDT15 genes are both related to mercaptopurine intolerance in acute lymphoblastic leukaemia patients from Uruguay. Br. J. Haematol. 181, 252-255 (2018).

27. Suzuki, H. et al. Genotyping NUDT15 can predict the dose reduction of 6-MP for children with acute lymphoblastic leukemia especially at a preschool age. J. Hum. Genet. 61, 797-801 (2016).

28. Tanaka, Y. et al. Susceptibility to 6-MP toxicity conferred by a NUDT15 variant in Japanese children with acute lymphoblastic leukaemia. Br. J. Haematol. 171, 109-115 (2015).

29. Wahlund, M. et al. The role of TPMT, ITPA, and NUDT15 variants during mercaptopurine treatment of swedish pediatric patients with acute lymphoblastic leukemia. J. Pediatr. 216, 150-157 (2020).

30. Wang, D.-S. et al. Childhood acute lymphoblastic leukemia mercaptopurine intolerance is associated with NUDT15 variants. Pediatr. Res. https://doi.org/10.1038/s41390-020-0868-8 (2020).

31. Relling, M. V. et al. Clinical pharmacogenetics implementation consortium guideline for thiopurine dosing based on TPMT and NUDT15 genotypes: 2018 update. Clin. Pharmacol. Ther. 105, 1095-1105 (2019).

32. Tsujimoto, S. et al. Diplotype analysis of NUDT15 variants and 6-mercaptopurine sensitivity in pediatric lymphoid neoplasms. Leukemia 32, 2710-2714 (2018).

33. Akiyama, S. et al. Long-term effect of NUDT15 R139C on hematologic indices in inflammatory bowel disease patients treated with thiopurine. J. Gastroenterol. Hepatol. https://doi.org/10.1111/jgh.14693 (2019).

34. Walker, G. J. et al. Association of genetic variants in NUDT15 with thiopurine-induced myelosuppression in patients with inflammatory bowel diseasegenetic variants associated with thiopurine-induced myelosuppression in ibdgenetic variants associated with thiopurine-induced myelosuppression in IBD. JAMA 321, 773-785 (2019).

35. Tanaka, Y. et al. Interaction between NUDT15 and ABCC4 variants enhances intolerability of 6-mercaptopurine in Japanese patients with childhood acute lymphoblastic leukemia. Pharmacogenomics J 18, 275-280 (2017).

36. Moriyama, T., Relling, M. V. \& Yang, J. J. Inherited genetic variation in childhood acute lymphoblastic leukemia. Blood 125, 3988-3995 (2015).

37. Pui, C. H., Nichols, K. E. \& Yang, J. J. Somatic and germline genomics in paediatric acute lymphoblastic leukaemia. Nat. Rev. Clin. Oncol. 16, 227-240 (2019).

38. Diouf, B. et al. Association of an inherited genetic variant with vincristine-related peripheral neuropathy in children with acute lymphoblastic leukemia. JAMA 313, 815-823 (2015).

39. Kamdem, L. K. et al. Genetic predictors of glucocorticoid-induced hypertension in children with acute lymphoblastic leukemia. Pharmacogenet. Genom. 18, 507-514 (2008).

40. Karol, S. E. et al. Genetics of glucocorticoid-associated osteonecrosis in children with acute lymphoblastic leukemia. Blood 126, 1770-1776 (2015).

41. French, D. et al. A PAI-1 (SERPINE1) polymorphism predicts osteonecrosis in children with acute lymphoblastic leukemia: a report from the Children's Oncology Group. Blood 111, 4496-4499 (2008).

42. Karol, S. E. et al. Genetic risk factors for the development of osteonecrosis in children under age 10 treated for acute lymphoblastic leukemia. Blood 127, 558-564 (2016).

43. Fernandez, C. A. et al. HLA-DRB1 ${ }^{\star} 07: 01$ is associated with a higher risk of asparaginase allergies. Blood 124, 1266-1276 (2014).

44. Fernandez, C. A. et al. Genome-wide analysis links NFATC2 with asparaginase hypersensitivity. Blood 126, 69-75 (2015).

45. Witt, H. et al. Variants in CPA1 are strongly associated with early onset chronic pancreatitis. Nat. Genet. 45, 1216-1220 (2013).

\section{Acknowledgements}

The authors express their gratitude to all patients who participated in this study and their parents. The authors also acknowledge the efforts of the TPOG and the Childhood Cancer Foundation in Taiwan.

\section{Author contributions}

C.-H.Y. and Y.-L.Y. designed the study, analyzed data, and wrote the manuscript. C.-H.Y., D.-S.W., Y.-L.N. and S.-W.L. acquired and processed patient specimens and performed experiments. C.-Y.L., Y-H.C. L.R. and H.-Y C. performed the analysis. K.-H.L., S.-T.J., M.-Y.L., K.-H.W., H.-H.C., S.-W.C. and D.-T.L. provided clinical samples and data. The manuscript was written by C.-H.Y. and Y.-L.Y. and was reviewed and edited by all authors.

\section{Funding}

This work was supported by grants from the Ministry of Science and Technology, Taiwan (MOST-1032314-B-002-199- and MOST-107-2314-B-002-173-MY2 to YLY) and from the National Taiwan University Hospital (NTUH.105-N03 to YLY).

\section{Competing interests}

The authors declare no competing interests.

\section{Additional information}

Supplementary information is available for this paper at https://doi.org/10.1038/s41598-020-71468-y.

Correspondence and requests for materials should be addressed to Y.-L.Y.

Reprints and permissions information is available at www.nature.com/reprints.

Publisher's note Springer Nature remains neutral with regard to jurisdictional claims in published maps and institutional affiliations. 
(c) (i) Open Access This article is licensed under a Creative Commons Attribution 4.0 International cc) License, which permits use, sharing, adaptation, distribution and reproduction in any medium or format, as long as you give appropriate credit to the original author(s) and the source, provide a link to the Creative Commons license, and indicate if changes were made. The images or other third party material in this article are included in the article's Creative Commons license, unless indicated otherwise in a credit line to the material. If material is not included in the article's Creative Commons license and your intended use is not permitted by statutory regulation or exceeds the permitted use, you will need to obtain permission directly from the copyright holder. To view a copy of this license, visit http://creativecommons.org/licenses/by/4.0/.

(C) The Author(s) 2020 